\title{
Strukturelle Beziehungen zwischen pflanzlichen und tierischen Sekundärstoffen ${ }^{1}$
}

\author{
Structural Relations between Secondary Metabolites from Plants and Animals
}

\author{
Adolf Nahrstedt
}

Institut für Pharmazeutische Biologie der Technischen Universität Braunschweig, Bundesrepublik Deutschland

\begin{abstract}
A series of structurally identical or related compounds has been detected in plants and animals, especially in the arthropods. Being simple but also complex structures of terpenoids, alkaloids and some glycosides, these compounds must be regarded as secondary products. Some of these compounds are sequestered by animals from the plants but identical or closely related structures are also produced by animals. In many cases it has not been proven convincingly whether these compounds are sequestered or self synthesized by the animals. The present paper gives an overview on these substances also regarding their biological significance for animals.
\end{abstract}

\section{Einleitung}

Noch im Jahre 1921 konnte der Pflanzenphysiologe CZAPEK in seinem viel beachteten Werk „Biochemie der Pflanzen" schreiben, daß keine Sekundärstoffe in Tieren gefunden worden seien [1]. Diese Feststellung erstaunte wenig, betrachtete man doch zu dieser Zeit pflanzliche Sekundärstoffe als abgelagerte Abfallprodukte, während Tiere dagegen über wirkungsvolle Ausscheidungsmechanismen verfügen. Erst in der Mitte unseres Jahrhunderts wurde vor allem durch Arbeiten von Butenand, Eisner, Karlson, Reichstein, Rothschild und SCHILDKNECht sowie zahlreiche ideelle Anstöße von MotHES klar, daß tierische Sekundärstoffe nicht nur existieren, sondern daß ihnen auch deutliche Funktionen zukommen [2]. In vielen Fällen besteht erstaunliche strukturelle Úbereinstimmung mit pflanzlichen Sekundärstoffen [3], jedoch ist bei vielen Untersuchungen nicht schlüssig geklärt, ob das Tier durch Aufnahme oder durch Eigensynthese zu diesen Substanzen kommt. Unter Beachtung dieses Aspektes soll die folgende Über-

1 Vortrag anläßlich der 29. Vortragstagung der Gesellschaft für Arzneipflanzenforschung in Marburg, Juni 1981 sicht niedermolekulare, strukturgleiche und strukturähnliche Sekundärstoffe in Pflanzen und Tieren behandeln, wobei im wesentlichen einer Gliederung nach der chemischen Struktur gefolgt werden soll.

\section{Monoterpene}

Die Arbeiterinnen der Honigbiene besitzen am hinteren Segment ihres Hinterleibes eine Drüse, die nach ihrem Entdecker als Nassanoff-Drüse bezeichnet wird. Die Bienen produzieren darin bereits wenige Tage nach Beendigung ihrer Entwicklung zum fliegenden Insekt vier acyclische, biogenetisch nahe verwandte Monoterpene $[4,5,6]$,

(1) Monoterpene in der Nassanoff-Drüse von Bienen (Apis mellifica)

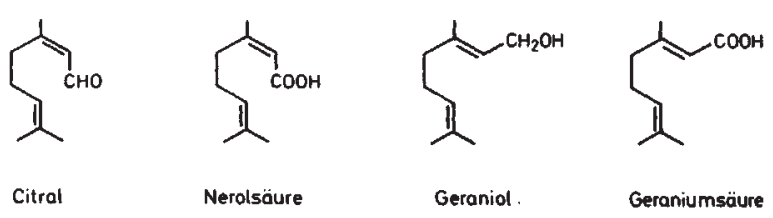

nämlich Geraniol und Geraniumsäure, das Isomerengemisch Citral und Nerolsäure (Abb. 1). Sämtliche Verbindungen sind aus dem aetherischen ÖI vieler höherer Pflanzen ebenfalls bekannt.

Die vier Monoterpene haben Pheromonfunktion; sie dienen zur Lokalisation von Nestern, zur Markierung von Futterquellen und sogar zur Markierung der Königin in einem Bienenschwarm. In seiner Geruchsqualität hat das Nassanoff-Sekret große Ähnlichkeit mit dem aetherischen Öl der Melisse, in dem Citral und Geraniol unter anderen als Hauptkomponenten enthalten sind [6]. Bienenschwärme lassen sich deshalb sowohl mit dem Öl als auch mit synthetischen Gemischen in jede gewünschte Richtung leiten [6].

Etliche Ameisen synthetisieren ebenfalls Monoterpene, die ihnen als Alarm- und Wehrsubstanzen dienen und die sie bei Gefahr aus Kopf- oder anderen Körperdrüsen sekretieren (Abb. 2). 
Monoterpene<smiles>C=CC(C)=CCC(C)C</smiles>

Amitermes evuncifer (Termiten) z. B. Lamiaceae

cis-B-Oeimen
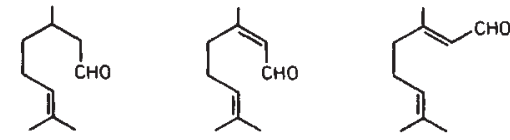

Acanthomyops claviger (Schuppenameisen) z.B. Rutaceae, Poaceae

Citronellat Citral

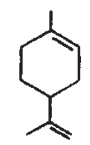

Myrmicaria natalensis (Knotenameisen) z.B. Rutaceae

Limonen

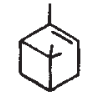

Nasutitermes spec. (Termiten)

Pinus spec. (Pinaceae)

$\alpha-P$ inen

Citral-Isomere und Citronellal werden von Acanthomyops claviger über Mevalonsäure aufgebaut [7, 8]. Vertreter der Knotenameisen produzieren das zyklische Monoterpen Limonen [9]. Blattschneiderameisen aus der Gattung Atta, z.B. Atta sexdens, besitzen in ihren Mandibulardrüsen $\beta$-Pinen, das Sesquiterpen Farnesol und den gesamten Monoterpensatz, der in den Nassanoff-Drüsen der Bienen vorkommt [10]. Auch Termiten benutzen solche Monoterpene als Abwehrsubstanzen, die auch in Pflanzen verbreitet sind. Die Soldaten von Amitermes evuncifer wehren sich gegen räuberische Ameisen, indem sie ihnen Wunden am Kopf beibringen und in diese Mono- und Sesquiterpen-haltige Wehrsekrete sezernieren, u.a. mit großen Mengen cis- $\beta$-Ocimen [11]. Die Ameisen wehren sich dagegen, indem sie Dimethylallyl-Pyrazine versprühen [11]: der Krieg mit chemischen Waffen ist also nicht erst vom Menschen erfunden worden.
Ein Vertreter der echten Borkenkäfer, Ips confusus, nutzt die Monoterpene in den Rinden von Pinaceen, um seine Futterstelle zu finden. Die pflanzlichen Monoterpene $\alpha$-Pinen und Myrcen haben hier zunächst die Funktion von Kairomonen. Einmal gelandet benutzt der Käfer die pflanzlichen Monoterpene zur Produktion von Pheromon- und Abwehrstoffen [ausführl. Darst. in 12]. So oxidiert er mit Hilfe eines im Darm angesiedelten Bakteriums $\alpha$-Pinen zu Verbenol (Abb. 3), ein Monoterpen, das auch im Pflanzenreich vereinzelt vorkommt [13].

Linalool wurde kürzlich in den Duftdrüsen einer Feuerwanze nachgewiesen [14] und $\beta$-Fenchon in den flüchtigen Sekreten einer Fruchtfliege [15] (Abb. 3). In beiden Fällen ist es nicht sicher, ob diese Substanzen aus Pflanzen stammen; die Fruchtfliege wurde auf Fencholfreier Nahrung gezogen, was ein Hinweis auf Eigensynthese sein könnte.

Stärker abgeleitete Monoterpene vom Iridoid-Typ wurden zuerst in Drüsenameisen der Gattung Iridomyrmex gefunden (Abb. 4). Iridodial [9] als namengebende Substanz, Chrysomelidial [16] und deren Cyclisierungsprodukte Iridomyrmecin [9] und Plagiolacton [16] wurden zwar nur in Ameisen und Chrysomelidenkäfern als Bestandteile der Wehrsekrete nachgewiesen, Pflanzen bilden jedoch ähnliche Lactone wie z.B. das Nepetalacton, bei denen aber grundsätzlich die Carbonylfunktion in Position 1 steht. Iridodial selbst wird als Zwischenprodukt der pflanzlichen Iridoidsynthese wieder verstärkt diskutiert $[17,18]$.

In den Wehrsekreten von zwei räuberischen Käfern aus der Familie der Staphyliniden ist neben Iridodial und Citronellal Actinidin (Abb. 4) gefunden worden [19], das als einfachstes Monoterpenalkaloid auch in Pflanzen vorkommt [Übersicht in 20].

\section{Sesqui- bis Sesterterpene}

Abbildung 5 zeigt einen Ausschnitt aus dem Spektrum höherer Terpene, die Tiere und Pflanzen gemeinsam führen. Lineare Sesqui- und Diterpene kommen in Ameisen und Bienen vor [21] und scheinen offensichtlich von den Tieren selbst synthetisiert zu werden.
(3) Monoterpene

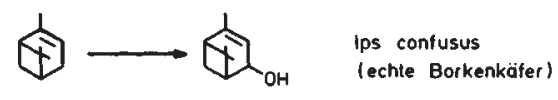

$\alpha-$ Pinen cis-Verbenol

Dysdercus intermedius

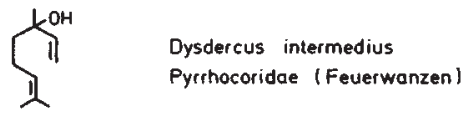

Linalool

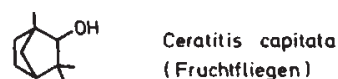

Fenchol
(Fruchtfliegen)

(4)

Iridoide Monoterpene

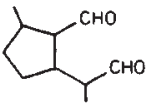

Iridodial

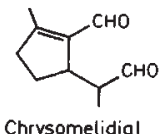

Chrysomelidial

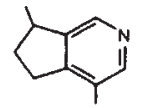

Actinidin

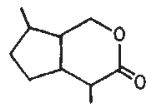

2.B. Ifidomyrmex humilis (Drusenameisen)

Iridomyrmecin

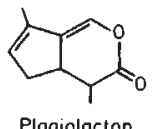

Plagiclacton

Hesperus semirufus

Philonthus politicus (Coleoptera, Käfer)

z.B. Actinia polygama (Actinidiaceae)
Plagiodera versicolora (Blattköfer)

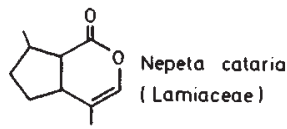

Nepetalacton 
(5) Sesquiterpene, Diterpene, Sesterterpene

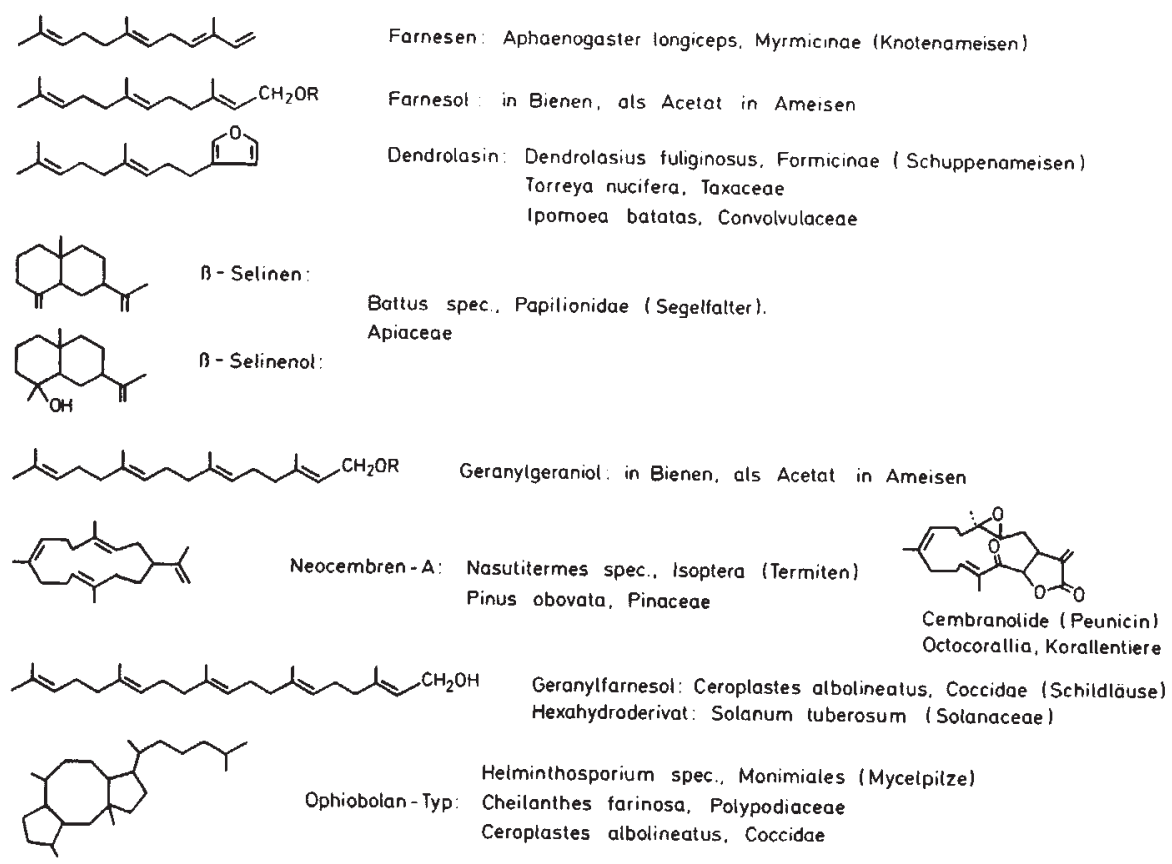

Das furanoide Sesquiterpen Dendrolasin wurde zunächst im Alarm- und Wehrsekret der Holzameise Dendrolasius gefunden, später dann auch in einer Taxacee, im Fuselöl der Süßkartoffel und schließlich aus einem primitiven Angehörigen des Tierreiches, aus dem Schwamm Oligoceras isoliert [22]. Das um eine C-5Einheit ärmere Perillen kommt ebenfalls in Dendrolasius vor [23] aber auch in der Lamiacee Perilla citrodosa [13].

Zwei Sesquiterpene aus der Eudesman-Reihe, $\beta$-Selinen und $\beta$-Selinol wurden in den Raupen eines Segelfalters gefunden [23]. Ein Eudesmen-1,6-diol, das Platambin, stammt aus dem Wehrsekret des Schnellschwimmer-Käfers Platambus maculatus [10]. Während Selinen und Selinol in Apiaceen verbreitet sind, kommt Platambin im Pflanzenreich nicht vor.

Bemerkenswert sind Diterpene vom Typ der Cembrane. Cembren A und seine Isomeren sind in den Kopfdrüsen von Termiten enthalten. Sie dienen den Soldaten von Nasutitermes und Cubitermes als Wehrsekret und Pheromon zur Wegemarkierung [24]. Dieselben Substanzen sind Bestandteile der Harzfraktion von Pinaceen, z.B. Pinus obovata $[23,25]$. Oxidierte Cembrene, vornehmlich mit Lactonstruktur und exocyclischer Doppelbindung in Konjugation zum Lactoncarbonyl, die Cembranolide, sind in bestimmten Korallen nachgewiesen worden. Es wird vermutet, daß die Gruppe der Octocorallia auf Grund dieser allgemein toxischen Verbindungen, die sie in ihre Umgebung exkretieren, den harten Konkurrenzkampf im Korallenriff glänzend bestehen [26].

Sesterterpene stellen eine noch recht kleine Gruppe der Terpenoide dar. Das lineare Geranylfarnesol ist im Wachs der Schildlaus Ceroplastes, als Hexahydroderivat

auch in der Kartoffel enthalten [27]. Sesterterpene vom Ophiobolan-Typ kommen ebenfalls in der Schildlaus, aber auch in Pilzen der Gattung Helminthosporium und in dem Farn Cheilanthes, einer Polypodiacee, vor [27].

\section{Steroide}

Die genannten Beispiele zeigen, daß Terpenoidsynthese zumindest in Ameisen, Bienen, Termiten und einigen Käfern ablaufen kann und dabei auch zu Produkten führt, die mit solchen aus dem Pflanzenreich z.T. strukturidentisch sind. Viele niedere Tiere sind jedoch nicht in der Lage, Triterpene vom Sterol-Typ zu synthetisieren. Hierzu gehören praktisch alle Insekten, Crustaceen, Vertreter der Mollusken, der Nematoden, der Protozoen [9]. Diese Tiere sind auf die Versorgung mit Steroiden aus Pflanzen angewiesen. Pflanzliche Steroide werden dabei entweder unverändert genutzt oder in der Regel über Cholesterin - im tierischen Stoffwechsel metabolisiert $[9,28,30]$.

Ecdysone sind für die Metamorphose von Insekten und Crustaceen notwendige Steroidhormone. Ihnen ist ein vicinal dihydroxylierter Ring A, eine mehrfach hydroxylierte Seitenkette und eine Ketofunktion sowie eine Doppelbindung in Ring B gemeinsam [Übersicht bei 30]. Seit Mitte der 60er Jahre findet man jedoch auch bei Pflanzen in zunehmendem Maße Steroide mit allen chemischen und physiologischen Eigenschaften der Ecdysone. Abb. 6 zeigt zwei Beispiele: $\alpha$-Ecdyson und Ecdysteron wurden aus dem Seidenspinner und dem Adlerfarn isoliert, Ecdysteron zusätzlich aus einer Taxacee und einer Languste. Während die Bedeutung der Ecdysone für die Tiere weitestgehend klar ist, existieren keine sicheren Erkenntisse über deren eventuelle Bedeutung für Pflanzen [29, 30, 31]. 
Ecdysone + Vitamin D
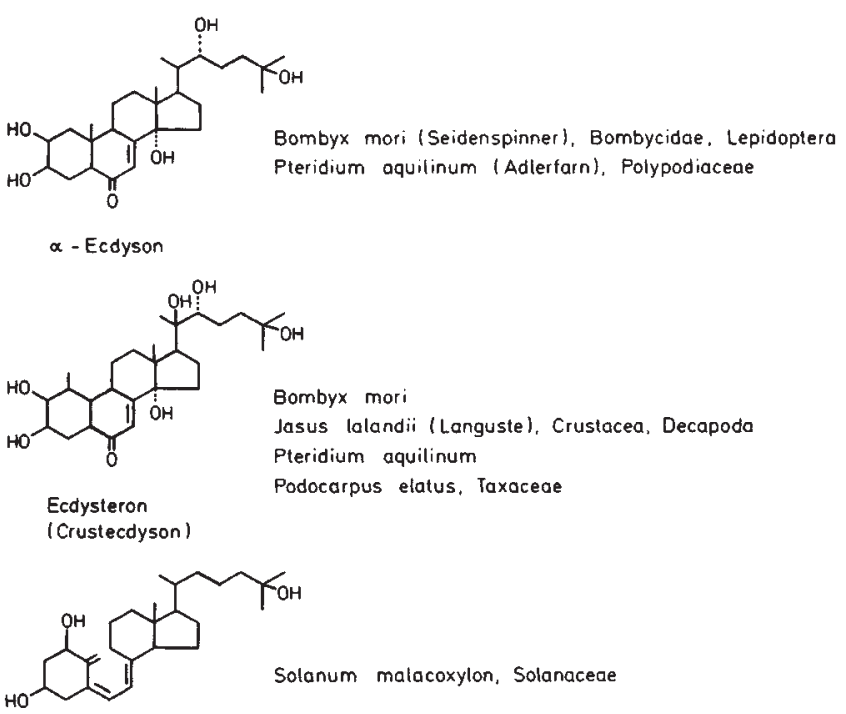

1,25-Dihydroxyvitamin $D_{3}$ als Glykosid

Ein anderes, erratisch in Pflanzen vorkommendes Steroid, dessen biologische Bedeutung für Pflanzen ebenfalls unbekannt ist, ist Vitamin D-3 [32]. In Solanum malacoxylon ist sogar ein Glykosid (MG über 1000) des 1,25-Dihydroxycholecalciferols (Abb. 6) gefunden worden [33]; dieses physiologisch aktive Vitamin D-3 wird sonst nur in den Nieren von Säugern, auch des Menschen, gebildet.

Seit den bahnbrechenden Arbeiten von RoThSCHILD und Reichstein gehört es zum Lehrbuchwissen, daß Schmetterlinge aus der Familie der Scheckenfalter (Nymphalidae), wie z.B. Danaus plexippus, Herzglykoside enthalten können, wenn sie im Raupenstadium auf bestimmten Asclepiadaceen gefressen haben [34, 35, $36,37]$.

\section{(7) Herzglykoside}
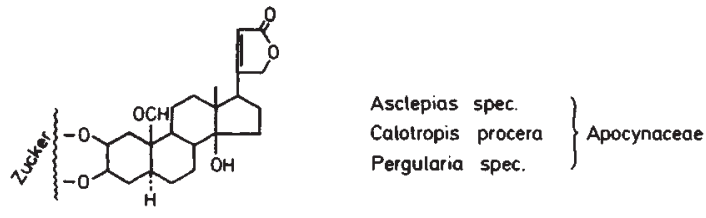

Calotropagenin-Glykoside

Pergularia spec.

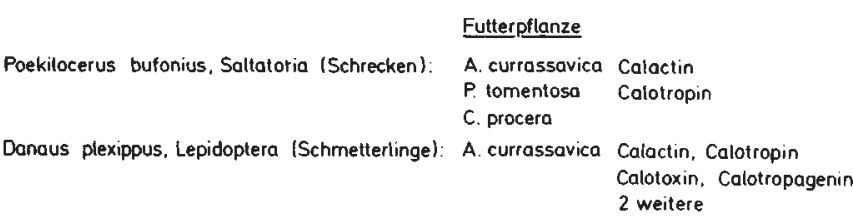

Die Struktur dieser aus Asclepiadaceen stammenden ziemlich Hydrolyse-stabilen Glykoside weicht von derjenigen der therapeutisch genutzten ab: Ring A und B sind trans verknüpft, und der Zuckerpartner ist über 2 Hydroxylgruppen mit dem Aglykon verbunden (Hemi- ketal an C-2). Die Glykoside werden von den Raupen selektiv gespeichert und in wesentlich größerer Menge akkumuliert, als sie in den pflanzlichen Organen enthalten sind: die Anreicherung kann bis zum 10-fachen der Konzentration im pflanzlichen Gewebe gehen [37]. Der Vorteil für den Schmetterling besteht darin, daß er durch Glykoside wie Calactin und Calotropin für Vögel ungenießbar wird:

Sie müssen nach dem Verspeisen eines solchen Falters heftig erbrechen und meiden in Zukunft die auffällig und typisch gemusterten, aposematischen Schmetterlinge [102]*. Der chemische Schutz ist so effektiv, daß andere Schmetterlinge im Verlauf ihrer Entwicklung sowohl Substanzen wie auch warnendes Farbmuster erworben haben (Müllersche Mimikry), andere sich nur mit dem Farb- und Formmuster begnügen (Bates'sche Mimikry) [38]. Es sei am Rande erwähnt, daß Herzglykosid-freie Schmetterlinge (Danaus, Euploea), die auf Herzglykosid-freier Nahrung gezogen wurden, trotzdem cardioaktive Substanzen mit z.Z. unbekannter Struktur besitzen [39].

Der Heuschreck Poekilocerus frißt ebenfalls auf Asclepiadaceen; er kumuliert nur zwei von mindestens 7 der in den Futterpflanzen enthaltenen Glykoside (Abb. 7) [37] und sezerniert sie mit einem Sekret, das in Abwehrstellung als Schaum auf beiden Körperseiten austritt [34]. Einmal gespeicherte Cardenolide können bei Herzglykosid-freier Nahrung bis auf die F-2-Generation weitergegeben werden [36], ein Beispiel für die enorme Speicherökonomie dieser Tiere.

(8) Herzglykoside

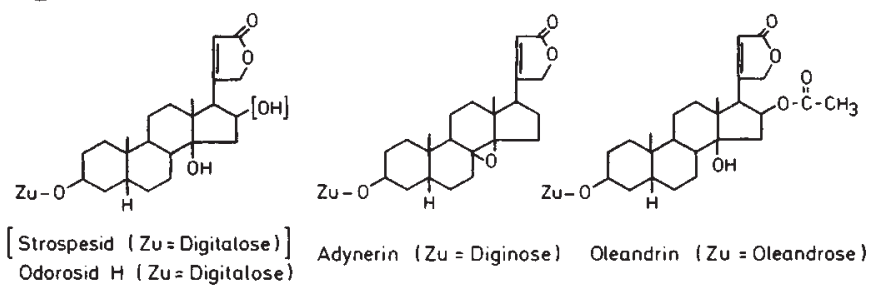

Herzglykoside, die mit den therapeutisch verwendeten strukturgleich sind, gibt es in Aphiden (Blattläuse), die sie mit dem Phloemstrom aus z.B. Nerium oleander selektiv aufnehmen (Abb. 8). Es scheint, daß zumindest monophage Blattläuse allgemein gute Speicherer sekundärer Pflanzenstoffe sind, sofern diese mit dem Phloemstrom transportiert werden: Aphiden auf Papaver somniferum enthalten Morphin [40], solche auf Bit-

* Vögel können jedoch lernen, die Cardenolid-Abwehr der Schmetterlinge zu überwinden. [Nature 291, 67 (1981)] 
(9)
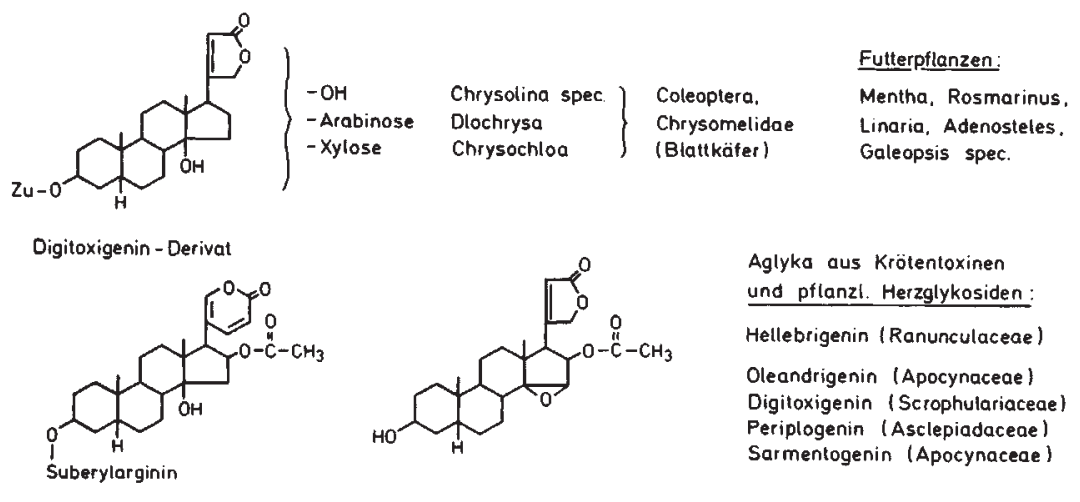

Aglyka aus Krötentoxinen und ptlanzl. Herzglykosiden: Hellebrigenin (Ranunculaceae) Oleandrigenin (Apocynaceae) Digitoxigenin (Scrophulariaceae) Periplogenin (Asclepiadaceae)

Bufotoxin aus Bufo bufo Bufonidae (Kröten)

Aglykon aus B. gargorizans (Ch'an Su)

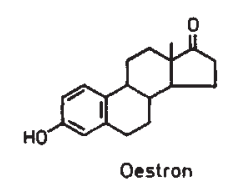

Phoenix dactylifera, Arecaceae Prunus armeniaca, Rosoceae
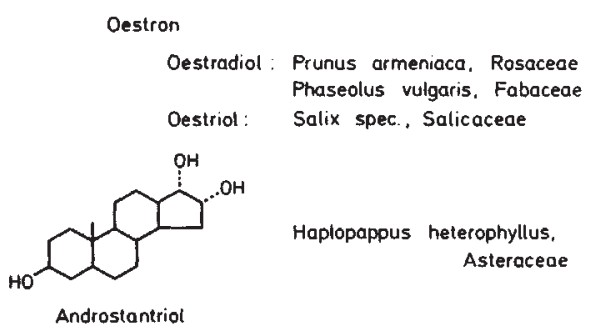

Haplopappus heterophyllus. Asteraceae

Saponine in Stachelhäutern (Echinodermata)

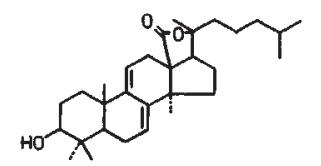

Holothurioidece (Seegurken) Asteroideae (Seesterne)
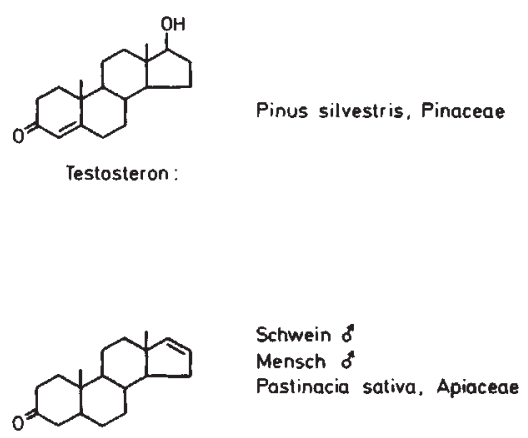

5a-androst-16-en-3-on ("boar taint steroid")

Seychellogenin

ter-Lupinen enthalten Chinolizidine [41]; unter diesem Aspekt ist es empfehlenswert, bei der Bearbeitung von Sekundärstoffen in Pflanzen sich auch deren Blattläuse näher anzusehen. Marienkäfer, die Herzglykosid-haltige Blattläuse fressen, akkumulieren damit ebenfalls Herzglykoside [42]. Auch die Lygaeiden, Blattwanzen, nehmen Herzglykoside auf und speichern sie. Über eine evtl. ökologische Bedeutung dieser Verbindungen für Blattwanzen und -läuse ist nichts bekannt.

Verschiedene Blattkäfer (Chrysomelidae) dagegen synthetisieren ihre Herzglykoside selbst. Obwohl sie auf Herzglykosid-freien Pflanzen im Larvenstadium fressen, enthalten sie in ihrem Wehrsekret DigitoxigeninGlykoside mit den sonst unüblichen Pentosen Arabinose und Xylose als Zuckerpartner (Abb. 9) [43].

Auch Kröten synthetisieren ausgehend von Cholesterin die in ihren Hautdrüsensekreten enthaltenen herzaktiven Verbindungen selbst [44, 45]. Die Übereinstimmung mit pflanzlichen Substanzen besteht nur noch im Aglykon (Abb. 9); bekannt sind Derivate mit einer
Cumalin-Substitution am C-17; weniger bekannt ist, daß auch pflanzentypische Aglyka mit einem Butenolid-Ring frei [46], als Suberoyl-Ester [47] oder als Schwefelsäure-Halbester [48] in Kröten enthalten sind.

Schließlich soll kurz auf Struktur-Ähnlichkeiten und Übereinstimmungen bei Östrogenen und Androgenen hingewiesen werden. Oestron, Testosteron, Androstantriol (Abb. 10), die bei Tieren unerläßliche Hormonfunktionen erfüllen, kommen auch in Pflanzen zwischen $0,1-15 \mathrm{mg} / \mathrm{kg}$ vor, ohne daß man bislang eine Funktion dieser Verbindungen für die Pflanzen erkannt hätte [31].

Seesterne und Seegurken produzieren Steroidsaponine vom Lanostan-Typ (Abb. 10), meist 18,20-Lactone mit starker hämolytischer Aktivität [45, 49, 50, 51]. In Pflanzen wurde dieser Saponintyp noch nicht gefunden.

Als Kuriosität sei am Rande erwähnt, daß ein wichtiges Kontaktpheromon des männlichen Schweines [52], das 5a-Androst-16-en-3-on, auch im Achselschweiß des männlichen Menschen [52] und im Pastinak und 
Sellerie vorkommt [53]. Über die Bedeutung dieses " boar taint steroid" in Pflanzen und Menschen werden keine Angaben gemacht.

\section{Carotinoide}

Sexualhormone und Saponine werden in den sie führenden Organismen jeweils selbst gebildet. Carotinoide dagegen, die in vielen Insekten [54], aber auch in Fischen [55] und Vögeln eine ökologische Funktion als Farbsignalgeber [56] erfüllen, stammen grundsätzlich aus Pflanzen und werden nur z.T. metabolisiert. Hierzu ein Beispiel (Abb. 11):

\section{Carotinoide}

\begin{tabular}{|l|c|c|c|}
\hline$\%$ & $\begin{array}{c}\text { Brassica } \\
\text { oleracea }\end{array}$ & $\begin{array}{c}\text { Pieris brassicae } \\
\text { (Puppen) }\end{array}$ & $\begin{array}{c}\text { Apanteles glomeratus } \\
\text { (Puppen) }\end{array}$ \\
\hline$\alpha$-Carotin & - & - & - \\
B-Carotin & 27.9 & 54.3 & 64.4 \\
$\begin{array}{l}\text { 5.6-Monoepoxy- } \\
\alpha \text {-Carotin } \\
\text { 5.6-Monoepoxy- } \\
\text { B-Carotin }\end{array}$ & Spuren & 1.7 & 4.7 \\
Violaxanthin & 15.3 & 6.8 & 5.4 \\
Lulein & 39.0 & - & - \\
Zeaxanthin & - & 37.2 & 25.5 \\
Neoxanthin & 17.1 & - & - \\
\hline
\end{tabular}

Untersuchungen aus dem Arbeitskreis von RothsCHILD haben gezeigt, daß die Carotinoidausstattung der Kohlblätter von der darauf fressenden Raupe des Kohlweißlings quantitativ und qualitativ verändert in die Puppe des Schmetterlings übernommen wird. Eine in der Kohlweißlingspuppe schmarotzende Schlupfwespe übernimmt die Carotinoide in ihrer quantitativen $\mathrm{Zu}$ sammensetzung fast unverändert [57]. Auch hier nehmen, wie im System Apocynaceen $\rightarrow$ Blattläuse $\rightarrow$ Marienkäfer (s.v.), die Sekundärstoffe an der Verlängerung der Nahrungskette teil.

N-haltige Verbindungen

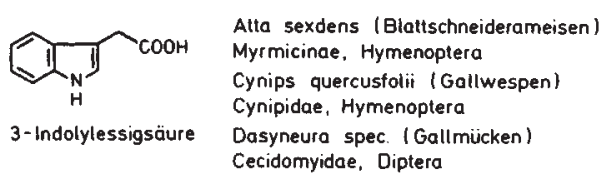

$$
\begin{aligned}
& \mathrm{CH}_{3}-\mathrm{O}-\mathrm{C}-\mathrm{O}-\mathrm{CH}_{2}-\mathrm{CH}_{2}-\mathrm{N}=\mathrm{CH}_{3} \mathrm{CH}_{3} \text { Arctia caja. Lepidoptera (Schmetterlinge) Moraceae } \\
& \mathrm{CH}_{3} \text { Zygaena spec., ". Scrophulariaceae } \\
& \text { Acetylcholin } \\
& \text { Brassieaceae }
\end{aligned}
$$

$\mathrm{CH}_{3}>\mathrm{C}=\mathrm{CH}-\stackrel{\mathrm{O}}{\mathrm{C}}-\mathrm{CH}_{2}-\mathrm{CH}_{2}-\mathrm{N}=\mathrm{CH}_{\mathrm{CH}_{3}}^{\mathrm{CH}_{3}}$ Arctia caja

B, B-Dimethylacrylcholin

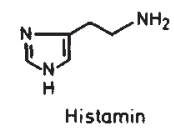

$$
\begin{aligned}
& \text { Arctio caja } \\
& \text { Zygaena spec. } \\
& \text { Laothoe populi, Lepidoptera }
\end{aligned}
$$

Seneciosäure 2.8 . in Apiaceae

Urticaceae

\section{$N$-haltige Verbindungen}

Die folgenden Verbindungen sind sämtlich Stickstoffhaltige Substanzen, von denen man wegen ihrer z.T. spezifischen und z.T. starken Wirkungen geradezu erwarten möchte, daß sie auch von Tieren in verschiedenster Weise genutzt werden.*

Blattschneiderameisen wie Atta sexdens leben mit Pilzkulturen zusammen, die sie regelmäßig mit frischen Blattstücken als Substrat versorgen. Darüberhinaus produzieren die Ameisen in ihren Metathorakaldrüsen ein Exkret, das $\beta$-Indolylessigsäure (Abb. 12) enthält, ein für höhere und niedere Pflanzen bedeutsamer Wuchsstoff. Die Ameisen sind offenbar in der Lage, durch exakte Dosierung von IES an die Pilze das Wachstum ihrer sogenannten Pilzgärten zu regulieren [10]. IES ist auch für Gallwespen und Gallmücken wahrscheinlich gemacht [10].

Die nicht proteinogene Aminosäure Azetidin-2-carbonsäure, ein Prolin-Analogon, kommt in verschiedenen Liliaceen, insbesondere Convallaria und Polygonatum vor [61]; kürzlich ist sie auch aus zwei Meeresschwämmen als antidermatophytisch wirkende Substanz isoliert worden [117].

Der braune Bärenspinner, Arctia caja, speichert im ganzen Körper Acetylcholin und Histamin (Abb. 12) [56], zwei Amine, die auch von Pflanzen zur Verteidigung genutzt werden, z.B. in den Brennhaaren mancher Urticaceen. $\beta, \beta$-Dimethylacrylcholin ist in den Wehrdrüsen des braunen Bärenspinners enthalten [56], eine Verbindung, die in Pflanzen nicht existiert [13], jedoch vom Schmetterling leicht aus Cholin und im Pflanzenreich verbreiteter Seneciosäure aufgebaut werden könnte.

Die Arctiiden (Bärenspinner) sind ein markantes Beispiel dafür, da $B$ manche Insekten sich nicht nur auf einen Sekundärstoff oder eine Sekundärstoffgruppe konzentrieren: In Arctia caja sind je nach Lebensbedingungen Herzglykoside, Amine, Pyrrolizidinalkaloide und - wenn man ihn auf Cannabis-Blättern zieht - auch Cannabinoide nachgewiesen worden $[56,58]$. Die Fähigkeit, verschiedene Stoffe zu akkumulieren und gleichzeitig zu nutzen, scheint besonders bei den Gliedertieren weit verbreitet zu sein und stellt die chemische Abwehr und chemische Kommunikation auf eine breite stoffliche Basis.

\section{Piperidinderivate}

Aus dem Wehrsekret der Feuerameisen ist ein Piperidinderivat, das Solenopsin A (Abb. 13) isoliert worden mit haemolytischen, phytotoxischen, insecticiden und antibiotischen Eigenschaften [59, 23]. Die Verbindung hat - bis auf konfigurative Unterschiede - große Ähnlichkeit mit den Cassia-Alkaloiden vom Typ des Cassins [60] und dem Pinidin aus Picea-Arten [61], das aus

* Alkaloide als Kondensationsprodukte von DOPA, Tyrosin oder Tryptophan mit Formaldehyd oder Acetaldehyd werden für Säugetiere als Stoffwechselprodukte diskutiert [Lloydia 37, 196 (1974)]. 
(13) Piperidinderivate

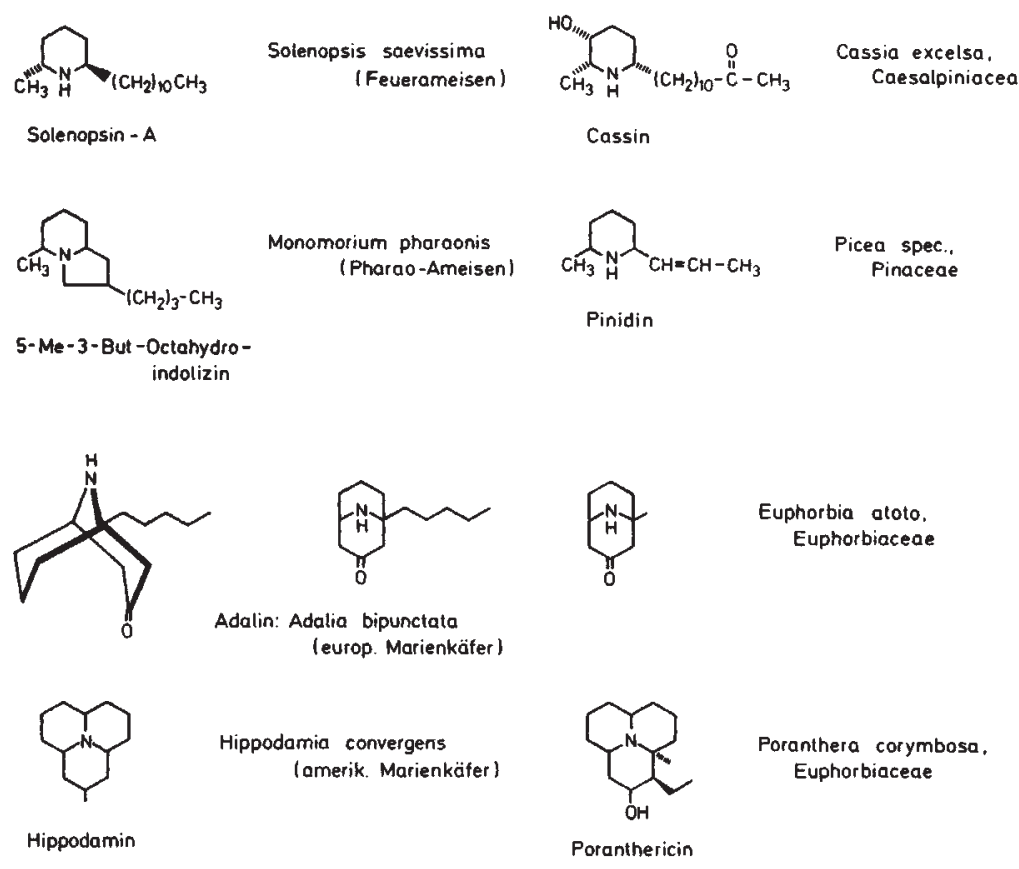

(14) Anabasein

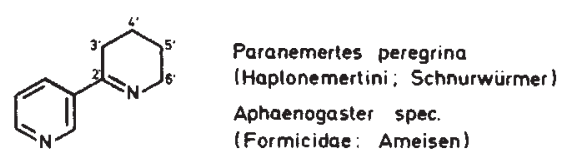

(Formicidae: Ameisen)<smiles>C1=CCC(c2cccnc2)NC1</smiles>

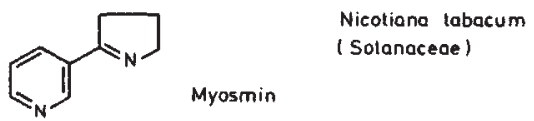

Nicotione tabacum (Solanaceae)
Acetat-Einheiten aufgebaut wird [62]. Es ist wahrscheinlich, daß das bicyclische 5-Methyl-3-butyl-octahydroindolizin, ein Pheromon aus der Pharaoameise, biogenetisch mit Solenopsin A verwandt ist.

Bicyclische und tricyclische Piperidinderivate, wie das Adalin [64], das Hippodamin [65] und sein N-Oxid, das Coccinellin, schützen wegen ihres bitteren Geschmakkes unseren europäischen Marienkäfer und seinen amerikanischen Verwandten (Abb. 13). Der Schutz ist offensichtlich so wirkungsvoll, daß die in jeder Hinsicht sehr anpassungsfähigen Schaben eine Mimikry-Form ausgebildet haben, die sich nur noch durch die langen Antennen vom Modell unterscheidet [38]. Bi- und tricyclische Marienkäfer-Alkaloide werden aus Acetat von den Käfern selbst aufgebaut [62]. Sehr ähnliche Strukturen - ebenfalls aus dem Polyketidstoffwechsel - kommen aber auch in Euphorbiaceen vor [66, 67], das Poranthericin in Poranthera corymbosa und ein namenloses bicyclisches System in Euphorbia atoto (Abb. 13).

Nur kurz soll auf das Pyridinalkaloid Anabasein hingewiesen werden, das bislang zweimal im Tierreich gefunden wurde $[68,69]$ und entgegen anders lautenden Behauptungen im Pflanzenreich nicht vorkommt. In $\mathrm{Ni}$ cotiana tabacum existieren jedoch Isomere bzw. Homologe, das Anatabin und das Myosmin (Abb. 14).

\section{Pyrazine}

Pyrazin-Derivate mit außerordentlich niedriger Geruchsschwelle werden von einigen Drüsen- und Stachelameisen als Alarm- und Abwehrsubstanzen genutzt (Abb. 15). Es handelt sich um 3-Alkyl-2-methoxypyrazine $[70,23]$. Ein strukturgleiches 3-Isobutyl-2-methoxypyrazin ist als Aromastoff in Capsicum annuum enthalten [71]. Die im fermentierten und gerösteten Kaffee
(15) Eyrazine

$$
\begin{aligned}
& \text { 3-Alkyl-2-methoxy- } \\
& \text { pyrazoline ( } \mathrm{R}=\text { iProp, iBut) } \\
& \mathrm{R}=\mathrm{iBut} \quad \begin{array}{l}
\text { Iridomyrmex humilis, Dolichoderinae (Drissenameisen) } \\
\text { Ondontomachus spec., Ponerinae (Stachelameisen) }
\end{array}
\end{aligned}
$$

und Kakao gefundenen Pyrazine besitzen keine 2-Methoxysubstitution $[71,72]$.

\section{Pyrrolizidin-Alkaloide}

Eine andere Gruppe pflanzlicher Alkaloide scheint für die Wechselbeziehungen zwischen Pflanzen und Insekten schon jetzt mindestens ebenso bedeutungsvoll geworden zu sein wie die Herzglykoside. Dies sind die insbesondere in Boraginaceen, Fabaceen, Asteraceen, aber auch in einigen Poaceen vorkommenden Pyrrolizidin-Alkaloide.

Abb. 16 zeigt die z.Z. in Schmetterlingen nachgewiesenen aus Pflanzen stammenden Pyrrolizidine. Sie werden interessanterweise von denselben Schmetterlingsarten akkumuliert, die auch Herzglykoside speichern [42]. Auf diese Beobachtungen stützen Pliske und Mitarbeiter ihre Theorie, daß die Vorläufer der heute den Schmetterlingen als Nahrungspflanzen dienenden Asclepiadaceen, Apocynaceen, Boraginaceen und Asteraceen ursprünglich zum Aufbau sowohl von Herzglykosiden als auch von Pyrrolizidinen fähig waren und diese Syntheseleistung im Verlauf der Evolution auf die mehr spezialisierten Nachfahren aufgespalten wurde [73]. Vereinzelt finden wir auch Pyrrolizidin-Alkaloide in rezenten Apocynaceen, z.B. Parsonsia-Arten. 
(16)

Pyrrolizidin-Alkaloide

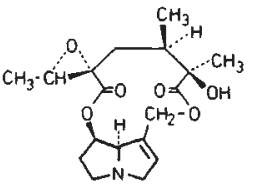

Jacobin (1)

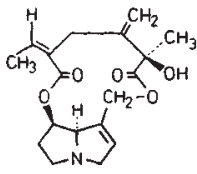

Seneciophyllin (V)

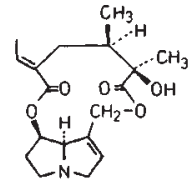

Senecionin (II)

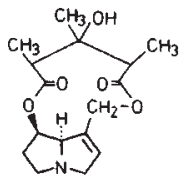

Crispotin (VI)

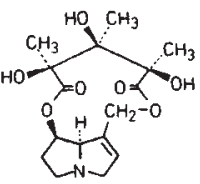

Monocrotalin (III)

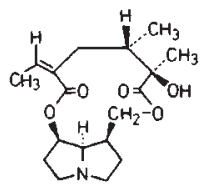

Rosmarinin (VII)

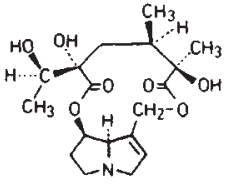

Jacolin (IV)

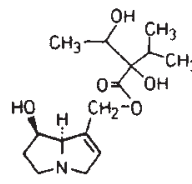

Lycopsamin (VIII)

\begin{tabular}{|ll|l|l|}
\hline Lepidoptera & Magnoliopsida & Alkaloide \\
\hline Arcliidae & Arctia caja & Senecio vulgaris (Asteraceae) & II. V. Integerrimin \\
& Tyria jacobea & Senecio vulgaris & I. II. IV. Jacozin. Integerrimin \\
& Amphicallia bellatrix & Crotolaria spec. (Fabaceae) & VI. Tricodesmin \\
& Argina cribaria & Crotolaria spec. & III \\
Nymphalidae & Danaus spec. & Parsonsia spec. (Apocynaceae) & VIII \\
(Scheckenfalter) & Euploea spec. & Parsonsia spec. & VIII \\
& Danaus chrysippus & Senecio plerophorus & II/V. VII \\
\hline
\end{tabular}

Verschiedene Bärenspinner und Scheckenfalter nehmen im Larvenstadium Pyrrolizidine und deren N-Oxide aus verschiedenen Futterpflanzen auf $[42,75]$ und speichern sie unverändert, häufig aber in anderer Relation zueinander als sie in den Futterpflanzen enthalten sind. Es handelt sich vornehmlich um solche Pyrrolizidin-Alkaloide, die alle Strukturmerkmale für eine stark alkylierende und damit cytotoxische Wirkung besitzen [76]: 1,2-Doppelbindung im Necin-System, Veresterung beider Hydroxylgruppen, macrocyclisches System der beteiligten Nec-Säuren. Derartige Pyrrolizidine sind in allen Lebensstadien der Schmetterlinge gefunden worden, und es ist plausibel anzunehmen, daß sie als Schutzsubstanzen dienen [77].

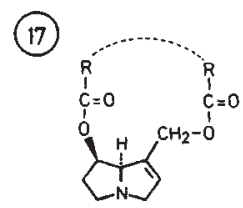

Asteraceae (Senecio, Eupatorium) Boraginaceae (Heliotropium. Fabaceae (Crofolaria)

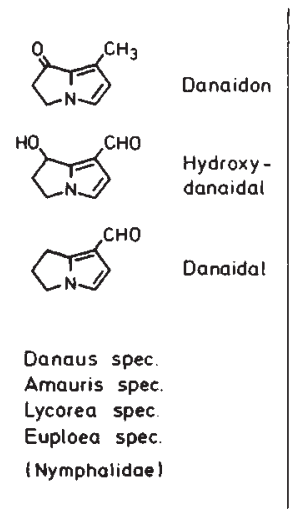

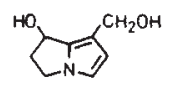

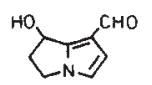

Utetheisa spec. (Arctiidoe) des Speichels Pyrrolizidine aufnehmen, die man später aus dem Falter in Form säurefreier Necine unterschiedlichen Oxidationsgrades isolieren kann [74, 79-83]. Die genuinen Pyrrolizidin-Ester werden bei dieser Transaktion in die in Abb. 17 aufgeführten nicht toxischen [76] Pyrrolizidine $-z . T$. ebenfalls als N-Oxide - überführt. Diese Verbindungen sind flüchtig und wichtige Bestandteile des Pheromonsystems der männlichen Falter. Ohne sie haben die Männchen keine Paarungschancen. Der Großteil dieser Pheromon-Pyrrolizidine ist in einem beim Hochzeitsflug ausstülpbaren Haarpinsel enthalten [83], über den sie auch an den umgebenden Gasraum abgegeben werden. Pyrrolizidinalkaloide sind die einzigen bekannten pflanzeneigenen Sekundärstoffe, die sowohl als Abwehrstoffe als auch metabolisiert als Sexualpheromone genutzt werden.

\section{Aristolochia-Säuren}

Aristolochia-Säuren, Nitrophenanthrenderivate, die von verschiedenen Aristolochiaceen stammen (Abb. 18 ), werden von einigen Segelfaltern im Raupensta-

(18)

Aristolochia-Säuren

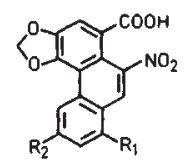

Jüngere Arbeiten aus dem Arbeitskreis von EISNER und Meinwald und auch Schneider in Seewiesen haben gezeigt, daß bevorzugt männliche Falter durch Einspeicheln von getrockneten Pflanzenteilen und Aufsaugen

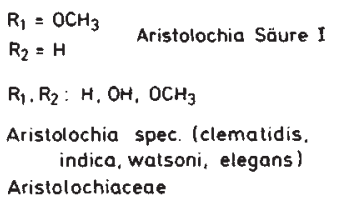

Papilionidae - Lepidoptera (Segelfalter)

Zerynthia polynexa (Puppen) Pachlioptera spec. (Falter) Battus spec. (Falter und Puppen) 
dium akkumuliert und zumindest bis in das Puppenstadium transferiert [42]. Diese Verbindungen sind kürzlich als potentiell carcinogen erkannt worden [101]. Sie schmecken aromatisch bitter und könnten deshalb eine Abschreckungsfunktion haben.

\section{Cycasin}

Ein hinsichtlich der metabolischen Potenz von Schmetterlingen interessanter Befund wurde mit einem weiteren Bärenspinner gemacht. Die Raupen von Seirarctia echo enthalten Cycasin (Abb. 19), wenn sie auf verschiedenen Cycadaceen fressen [84]. Das Hydrolyseprodukt N-Methylazoxymethanol (MAM) ist ein potentes Alkylierungsmittel und hochtoxisch. Cycasin ist konzentriert in der Hämolymphe und den Malpighischen Gefäßen (Abb. 19), die $\beta$-Glucosidase-frei sind und

Methylazoxymethanol - glykoside

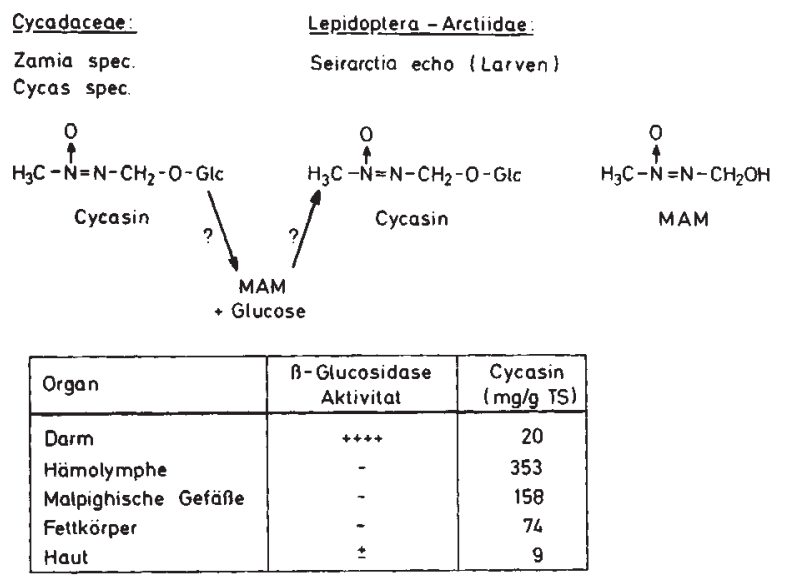

deshalb inerte Bereiche darstellen, in denen kein MAM freigesetzt werden kann. Wenn Pflanzenmaterial mit anderen, polareren MAM-Glykosiden als Cycasin verfüttert wird, ist trotzdem in den Raupen nur Cycasin nachweisbar. Der Autor, der diese Untersuchungen leider nicht weitergeführt hat, folgert deshalb, daß die im Darm vorhandenen Glucosidasen MAM-Glykoside zwar spalten, das Aglykon aber sofort wieder zu Cycasin glucosidiert wird und derartig inaktiviert in metabolisch inerte Räume der Raupe abgelagert wird [84]. Eine solche Reaktion stünde mit den in Insekten beobachteten Entgiftungsmechanismen für Fremdstoffe, zu denen auch Transglucosidierungen gehören, durchaus in Einklang [85].

\section{Cyanogene Glykoside}

Ein weiteres Kapitel soll eigenen Arbeiten zu diesem Thema gewidmet sein. Seit etwa 20 Jahren weiß man, $\mathrm{da} \beta$ das Widderchen, Zygaena filipendulae, Blausäure produziert, wenn man es verletzt [86]. Da die Tiere unverletzt keine Blausäure abgeben, sollte diese in irgendeiner Form fixiert sein.
Cyanogene Glykoside in Schmetterlingen (Lepidoptera)

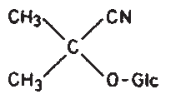

Linamarin

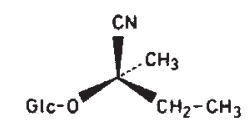

Lotaustralin

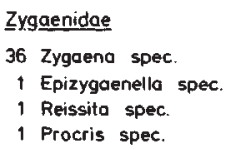

\section{Acraeinae \\ Heliconiinoe}

5 Acroeo spec.

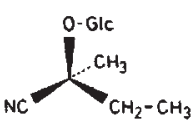

Epilotaustralin

2-3 Heliconius spec
Unsere Untersuchungen ergaben, daß zwei aus Pflanzen gut bekannte cyanogene Glykoside, Linamarin und sein Homologes Lotaustralin, nicht aber Epilotaustralin (Abb. 20), in Zygaena enthalten sind [87]. In den Zygaenidae scheint die Eigenschaft der Cyanglykosidführung weit verbreitet zu sein: 36 Zygaena Spec. und drei weitere nahe Verwandte enthalten beide Glucoside [88]. Im Verlauf unserer Arbeiten fanden wir noch zwei cyanogene Unterfamilien der Nymphalidae, die Acraeinae und Heliconiinae, die zwar selbst nahe miteinander verwandt sind, von den Zygaenen jedoch systematisch entfernt stehen. In beiden Unterfamilien sind ebenfalls Linamarin und Lotaustralin als cyanogene Verbindungen enthalten [89].

Viele Zygaenen fressen im Raupenstadium auf Papilionaceen, die ebenfalls Linamarin und Lotaustralin führen. Eine Aufnahme wäre also möglich. Viele Heliconiinen und Acraeinen dagegen fressen auf Passifloraceen, die vornehmlich einen anderen CyanglykosidTyp, nämlich solche mit Cyclopentenylrest enthalten. In diesem Fall wäre also eher eine de-novo Synthese in den Schmetterlingen zu erwarten.

Verfütterung von ${ }^{14} \mathrm{C}$-markiertem Valin und Isoleucin, die entsprechend der pflanzlichen Biogenese als Vorstufen für Linamarin und Lotaustralin in Frage kommen [90], an Zygaena und Heliconius-Raupen ergab Einbauraten von bis zu $10 \%$ in Linamarin und Lotaustralin [91, 92]. Damit wird die Synthesepotenz der Schmetterlinge für diese beiden Glucoside belegt.

Wir stehen hier vor der etwas verwirrenden Situation, daß Zygaenen dieselben Cyanglucoside selbst aufbauen können, die auch in ihren Futterpflanzen enthalten sind; Heliconius aber Linamarin und Lotaustralin aufbauen kann, obwohl er sich mit anderen Cyanglykosiden aus seiner Nahrung versorgen könnte. Wenn auch eine Aufnahme zumindest bei Zygaena nicht auszuschließen ist, zeigt dieses Beispiel doch, daß man selbst bei struktureller Identität der Stoffe in Tieren und deren Nahrungspflanzen nicht einfach auf eine Aufnahme schließen darf. 
Andererseits läßt sich mit Hilfe der phylogenetischen Stammbäume von Zygaenidae und Heliconiinae zeigen, $\mathrm{da} ß$ die Eigenschaft der Cyanglykosidführung ein recht ursprüngliches Merkmal ist [103, 104]. Bei einigen dieser Schmetterlinge ist auch eine hohe Kapazität zur Blausäure-Entgiftung beobachtet worden [86]. Es ist gut vorstellbar, daß diese Tiere mit der Fähigkeit, Blausäureglykoside zu bilden und zu speichern, auch die Fähigkeit zur Entgiftung des Hydrolyseproduktes Blausäure entwickelt haben und die letztere dieser beiden Fähigkeiten ihnen erst den Zugang zu Futterpflanzen mit cyanogenen Glykosiden eröffnet hat; bei den Heliconiinae die Passifloraceen, bei manchen Zygaenen die Papilionaceen, die nun zufällig auch Linamarin und Lotaustralin selbst bilden.

\section{Verschiedene Stoffe}

Nur stichwortartig sollen einige weitere bemerkenswerte Sekundärstoffe dieses Themas genannt werden (Abb. 21):

(21) Verschiedene Substanzen

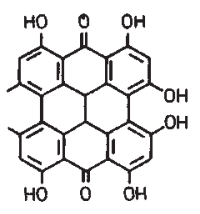

\section{Hypericin}

Hypericum hirsutum

(Hypericaceae)

Chrysolina brunsuicensis (Chrysometidae, Blattkäfer)

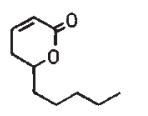

Massoilacton :
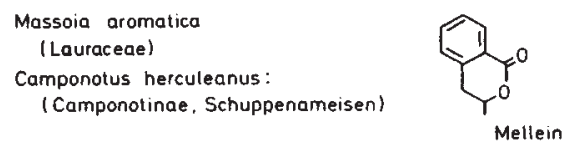

$\mathrm{CH}_{3}-\mathrm{CH}=\mathrm{CH}-\mathrm{C} \equiv \mathrm{C}-\mathrm{C} \equiv \mathrm{C}-\mathrm{CH}_{2}-\mathrm{CH}_{2}-\mathrm{COOH}$

8-cis-Dihydromatricaria-Säure

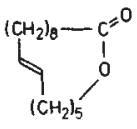
Hibiscus abelmoschus
(Malvaceae)

Ambrettolid

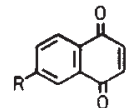

Tenebrionidce (Schworzkäfer)

6-Alkyl-Naphthochinone ( $R=$ Me. Et. Prop. But)

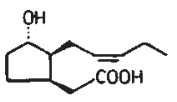

Cucurbinsäure (Cucurbita pepo)
Chouliognathus leconte (Cantharidae. Weichkäer) Asteraceae
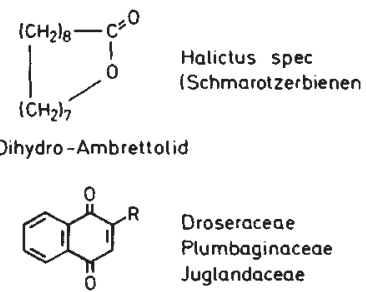

$\mathrm{R}=\mathrm{H} . \mathrm{CH}_{3}$ ester der Dihydromatricariasäure ist auch in Asteraceen enthalten. Möglicherweise nehmen die Käfer, die auf Compositen aggregieren, die Säure selbst oder Vorstufen dafür aus Compositen auf [93]. Der moschusartige Geruch von Hibiscusabelmoschus wird durch ein 16gliedriges, macrocyclisches Lacton hervorgerufen; sein Dihydroderivat ist in Schmarotzerbienen enthalten [94]. Viele Tembroiden, Schwarzkäfer, synthetisieren Naphthochinone über den Polyketidweg [95]. Sie unterscheiden sich von den pflanzlichen Naphthochinonen durch ihre Alkylsubstitution in Pos. 6. Prostaglandine, tierische Gewebshormone, sind kürzlich in der Rotalge Gracilaria lichenoides gefunden worden [96]. Ähnliche, verkürzte Strukturen, die Cucurbinsäuren, kommen in $\mathrm{Cu}$ curbitaceen vor [97].

(22) Glucosinolate, Senföle

$$
\begin{array}{ll}
\mathrm{CH}_{2}=\mathrm{CH}-\mathrm{CH}_{2}-\mathrm{C}^{\prime} \mathrm{S}-\mathrm{Glc} & \\
\text { Sinigrin } & \begin{array}{l}
\text { Pieris brassicae } \\
\text { (Kontweifling) } \\
\text { Lepidoptera }
\end{array} \\
\mathrm{CH}_{2}=\mathrm{CH}-\mathrm{CH}_{2}^{-}-\mathrm{NCS} & \mathrm{K}^{+} \\
\text {Allytisothiocyanat } & \text { Brassicaceae }
\end{array}
$$

Glucosinolate, insbesondere Sinigrin sind für viele Insekten toxisch [105]. Die Weibchen des Kohlweißlings (Pieris brassicae) jedoch werden durch Sinigrin (bzw. dessen Hydrolyseprodukt Allylisothiocyanat, Abb. 22) zur Eiablage auf Kohlblättern angeregt und die Raupen zum Fressen stimuliert [106]. Darüberhinaus werden Sinigrin und Allylsenföl in den Puppen des Kohlweißlings gespeichert, Allylsenföl allein wurde in Faltern und Eiern nachgewiesen [107].

\section{(23) Lignane}<smiles>COc1cc(CC2COC(=O)C2Cc2ccc(O)c(O)c2)ccc1O</smiles><smiles>COc1cc(CC(CO)C(C=O)Cc2cc(O)c(O)c(OC)c2)ccc1O</smiles>

Lignane (als Glucuronsaure konjugate) aus dem Urin von Menschen, Affen und Ratten<smiles>COc1ccc(CC2CCOC2Cc2ccc(OC)c(OC)c2)cc1OC</smiles><smiles>O=C(O)CC(CO)(Cc1ccc2c(c1)OCO2)Cc1ccc2c(c1)OCO2</smiles><smiles>CC(Cc1ccc(O)c(O)c1)Cc1ccc(O)c(O)c1</smiles>
Lignane aus höheren Pflanzen

Arctin (Arctium spec)
Nor-Dihydroguajaretsäure (Kreosolstrauch)
Auch die aus höheren Pflanzen bekannten Lignane wurden kürzlich als Glucuronsäurekonjugate, aber sonst den pflanzlichen Typen sehr ähnlich (Abb. 23), im Urin von Menschen, Ratten und Affen gefunden [98]. Es wird ein Zusammenhang zwischen der Gonadenfunktion und der Lignanausscheidung diskutiert [98]. Wehrsekretes eines Kanthariden-Käfers; der Methyl- worden, der auf Hypericum-Arten frißt [23, 42]. Masten, ist auch Bestandteil des Wehrsekretes von Schuppenameisen, neben Mellein, das man sich ebenfalls wie das Massoilacton gut als Acetogenin vorstellen kann [21, soilacton, in der Lauracee Cryptocarya massoia enthal- 
Marine Nahrungsketten

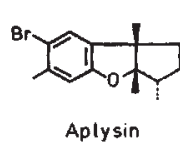

Aplysin
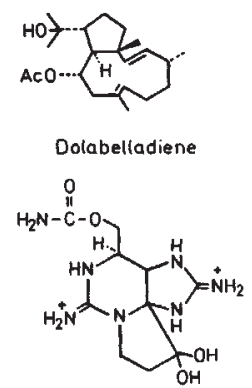

Saxitoxin

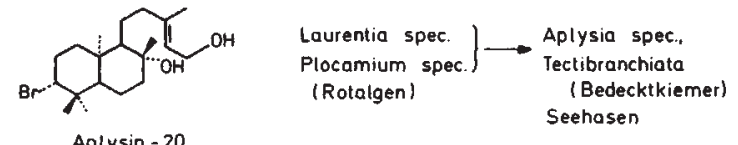

Aplysin -20

Glossophora galapagensis (Brounalgen) (Seehasen)

Gonyaulax catenello (Dinoflagellatce)

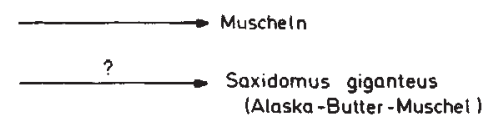

(Alaska-Butter-Muschel)

\section{Marine Nahrungsketten}

Die Weitergabe von Sekundärstoffen in Nahrungsketten ist im ökologisch hoch ausgenutzten marinen $\mathrm{Be}-$ reich weit verbreitet [108]. Abschließend soll deshalb nur auf wenige Strukturen beispielhaft hingewiesen werden:

Das Sesquiterpen Aplysin und die Diterpene Aplysin-20 und Dolabelladien (Abb. 24) werden von Rotbzw. Braunalgen produziert und von Seehasen, schnekkenähnlichen Meerestieren, mit den Algen gefressen und kumuliert. Die Schnecken sind dadurch für die Fraßfeinde so ungenießbar geworden, da $ß$ sie auf das sonst übliche Gehäuse verzichten können [99]. Berühmt-berüchtigt bei Muschelliebhabern ist das hochtoxische Saxitoxin (Abb. 24), ein Purinderivat und eines von mehreren Dinoflagellatentoxinen, die in Muscheln und Krabben angereichert werden [100].

\section{Schlußbetrachtung}

Über die vorgestellten Verbindungen hinaus sind verschiedene Pflanzensäuren in Käfern und Schmetterlingen $[109,110,111,112]$, Cyanhydrine in Tausendfüßlern [112] und Atropin unter bestimmten Bedingungen in einem Schmetterling nachgewiesen worden [113]. Gallenfarbstoffe kommen nicht nur als Abfallprodukte in Säugern, sondern mit spezifischen Funktionen auch in Pflanzen und niederen Tieren vor $[114,115]$.

Das gemeinsame Vorkommen identischer oder nahezu identischer Stoffe provoziert die Frage nach einer allgemeinen Bedeutung dieser Stoffe für die Organismen. Im allgemeinen wird dabei die Funktion als Abwehroder Lockstoff in den Vordergrund gerückt, Funktionen also, die das Zusammenleben der Organismen ordnen und ermöglichen und sich im Laufe der gemeinsamen Evolution herausgebildet und etabliert haben [z.B. 118, $119,120]$. Diese Funktionen werden bei Tieren akzep- tiert, sind aber bei Pflanzen nicht unumstritten. Akzeptiert man zumindest für einige stark wirksame Pflanzeninhaltsstoffe deren Abwehrfunktion (z.B. für Pyrrolizidine, cytotoxische Polyine, cyanogene Glykoside, Herzglykoside, Glucosinolate) dann wird deutlich, daß es einige Tiere verstanden haben, die pflanzlichen stofflichen Barrieren zu überwinden und sich darüber hinaus dieser Barriere in vielfältiger Weise selbst zu bedienen.

Die Strukturvielfalt ist im Pflanzenreich unvergleichlich stärker ausgeprägt. Tiere begnügen sich in der Regel mit einfacheren Strukturen und auch sequestrierende Insekten selektionieren und übernehmen häufig nicht den kompletten Satz einer pflanzlichen Stoffgruppe. Andererseits versetzt die Aufnahme- und Ablagerungsfähigkeit mancher Insekten immer wieder in Erstaunen: Der Grashüpfer Romalea microptera speichert in seinen Wehrdrüsen 2,5-Dichlorphenol, das wahrscheinlich von einem Herbicid stammt und mit der Nahrung aufgenommen wird [116].

Einige typische Pflanzeninhaltsstoffe sind in tierischen Organismen bisher (noch) nicht gefunden, wie z.B. die Flavonoide im weitesten Sinne. Doch ist sicher, daß die vorliegenden Arbeiten nur einen kleinen Einblick in die chemischen (und biochemischen) Übereinstimmungen zwischen Pflanzen und Tieren gestatten; einfach deshalb, weil noch viel zu wenig Daten vorliegen. Dieses Gebiet wird noch manche Uberraschung bieten, insbesondere bei den Insekten mit ihren engen ökologischen Beziehungen zur Pflanzenwelt.

\section{Danksagung}

Für anregende Diskussionen, Hinweise und Hilfe bei der Literatursuche danke ich sehr Dr. M. Boppré (Seewiesen), Dr. R. H. DAvIS (Ashford), Prof. K. W. Glombitza (Bonn), Prof. R. Hegnauer (Leiden), Prof. O. LARINK (Braunschweig), Prof. H. G. MAler (Braunschweig), Prof. C. Naumann (Bielefeld) und Prof. D. Schneider (Seewiesen). Mein Dank gilt ebenso Frau C. FORTH, die die zahlreichen Formelbilder anfertigte. 


\section{Literatur}

(1) Czapek, F.: Biochemie der Pflanzen, 2. Aufl. Vol. III, Jena 1921, G. Fischer.

(2) Mothes, K.: Wissenschaft und Fortschritt 30, 413 (1980).

(3) Mothes, K., in: Secondary Plant Products (E. A. Bell and B. V Charlwood, eds.), p. Iff, Berlin, 1980, Springer.

(4) Butler, C. G. and D. H. Calam: J. Insect Physiol. 15, 237 (1969).

(5) Boch, R. and D. A. Shearer: Nature 202, 320 (1964).

(6) Burgett, M.: Bee World 61, 44 (1980).

(7) Chaudha, M. S., T. Eisner, A Monro and J. Meinwald: J. Insect Physiol. 8, 175 (1962).

(8) Happ, G. M. and J. Meinwald: J. Amer. Chem. Soc. 87, 2059 (1965).

(9) Clayton, R. B. in: Chemical Ecology (E. Sondheimer and J. B. Simeone, eds.), p. 243, London, 1976, Academic Press.

(10) Schildknecht, H.: Angew. Chemie 88, 235 (1976).

(11) Baker, R., D. A. Evans and P. G. McDowell: Tetrahedron letters 4037 (1978).

(12) Harborne, J. B.: Introduction to Ecological Biochemistry, p. 98ff, London, 1977, Academic Press.

(13) Karrer, W.: Konstitution und Vorkommen der organischen Pflanzenstoffe, Basel, 1958, Birkhäuser; Ergänzungsband I, Basel 1977, Birkhäuser.

(14) Everton, I. J., D. W. Knight and B. W. Staddon: Comp. Biochem. Physiol. 63B, 157 (1979).

(15) Jacobson, M. and K. Okinata: Experientia 36, 629 (1980).

(16a) Meinwald, J., T. H. Jones, T. Eisner and K. Hicks: Proc. Natl. Acad. Sci. USA 74, 2189 (1977).

(b) Blum, M. S., J. B. Wallace, R. M. Duffield, J. M. Brand, H. M. Fales and E. A. Sokoloski: J. Chem. Ecol. 4, 47 (1978).

(c) Sugawara, F., K. Matsuda, A. Kobayashi and $\mathbf{K}$. Yamashita: $\mathbf{J}$. Chem. Ecol. 5, 635 (1979).

(17) Francis, M. J. O. in: Aspects of Terpenoid Chemistry and Biochemistry (T. J. Goodwin, ed.), p. 29, London, 1971, Academic Press.

(18) Inouye, H.: Planta med. 33, 193 (1978).

(19) Bellas, T. E., W. V. Brown and B. P. Moore: J. Insect Physiol. 20, 277 (1974).

(20) Jensen, S. R. and B. J. Nielsen: Bot. Notiser 128, 148 (1975).

(21) Blum, M. S. in: Biochemistry of Insects (M. Rochstein, ed.), p. 465ff, New York, 1979, Academic Press.

(22) Vanderah, D. J. and F. J. Schmitz: Lloydia 38, 271 (1975). (23) Rodriguez, E. and D. A. Levin in: Biochemical Interactions Between Plants and Insects (J. W. Wallace and R. L. Mansell, eds.), p. 214ff, New York, 1976, Plenum Press. (24) Wiemer, D. F., J. Meinwald, G. D. Prestwich and I. Miura: J. Org. Chem. 44, 3950 (1979).
(25) Hanson, J. R. in: Chemistry of Terpens and Terpenoids (A. A. Newman, ed.), p. 155ff, London 1972, Academic Press.

(26) Hadfield, M. G. and L. S. Ciereszko in: Drugs and Food from the Sea (P. N. Kaul and C. J. Sindermann, eds.), p. 145ff, 1978, The Univ. of Oklahoma.

(27) Cordell, G. A. in: Progr. in Phytochemistry Vol. IV (L. Reinhold, J. B. Harborne and T. Swain, eds.), p. 209ff, Oxford, 1977, Pergamon Press.

(28) Clayton, R. B.: J. Lipid Res. 5, 3 (1964).

(29) Rees, H. H. in: Aspects of Terpenoid Chemistry and Biochemistry (T. W. Goodwin, ed.), p. 181 ff, London, 1971, Academic Press.

(30) Karison, P.: Nova Act. Leopoldina 7 (Suppl.), 423 (1976).

(31) Geuns, J. M. C.: Phytochemistry 17,1 (1978).

(32) Heftmann, E. in: Progr. in Phytochemistry Vol. IV (L. Reinhold, J. B. Harborne, T. Swain, eds.), p. 257ff, Oxford, 1977, Pergamon Press.

(33) Wassermann, R., J. Henion, M. Haussler and T. McCain: Science 194, 853 (1976)

(34) Reichstein, T.: Naturwiss. Rdsch. 20, 499 (1967).

(35) Reichstein, T., J. v. Euw, J. A. Parsons and M. Rothschild: Science 161,861 (1968).

(36) Rothschild, M. in: Phytochemical Ecology (J. B. Harborne, ed.), p. Iff, London, 1972, Academic Press.

(37) Roeske, C. N., J. N. Seiber, L. P. Brower and C. M. Moffitt in: Biochemical Interactions Between Plants and Insects (J. M. Wallace and R. L. Mansell, eds.), p. 93ff, New York, 1976, Plenum Press.

(38) Wickler, W.: Mimikry, München, 1968, Kindler.

(39) Rothschild, M.: Nature 275, 649 (1978).

(40) Wichtl, M. pers. Mittlg.

(41) Wink, M.: pers. Mittlg., Publ. in Vorber.

(42) Rothschild, M. in: Insect Plant Relationships (H. v. Emden, ed.), p. 59ff, Oxford 1972, Blackwells Sci. Publ.

(43) Pasteels, J. M. and D. Daloze: Science 197,70 (1977).

(44) Siperstein, M. D., W. Murray and E. Titus: Arch. Biochem. Biophys. 67,154 (1957).

(45) Habermehl, G.: Gifttiere und ihre Waffen, Berlin, 1976, Springer. (46) Höriger, N., H. H. A. Linde und K. Meyer: Helv. Chim. Act. 53, 1503 (1970).

(47) Höriger, N., D. Zivanov, H. H A. Linde und K. Meyer: Helv. Chim. Act. 53, 2051 (1970).

(48) Fujii, Y., K. Shimada and T. Nambara: Chem. Ind. 614 (1976). (49) Tschesche, R. und G. Wulff: Fortschr. Chem. Org. Naturstoffe 30,461 (1973).

(50) Hiller, K. und G. Voigt: Pharmazie 32, 365 (1977).
(51) Anisimov, M. M. und V. J. Cirva: Pharmazie 35, 731 (1980). (52) Claus, R.: Acta endocr. (Khb) 225 (Suppl), 432 (1979).

(53) Claus, R. and H. O. Hoppen: Experientia 35, 1674 (1979).

(54) Feltwell, J. in: Biochemical Aspects of Plant and Animal Coevolution (J. B. Harborne, ed.), p. 277ff, London, 1978, Academic Press.

(55) Matsumo, T. and M. Katsuyama: Nippon Suisan Gakkaishi 45, 1533 (1979). Zit. nach CA 92:125 331.

(56) Rothschild, M. in: Coevolution of Animals and Plants (L. E. Gilbert and P. H. Raven, eds.), p. 20ff, Austin, 1975, Univ. Texas Press.

(57) Rothschild, M., G. Valadon and R. Mummery: J. Zool. Lond. 181, 323 (1977).

(58) Rothschild, M., M. G. Rowan and J. W. Fairbairn: Nature 266 650 (1977).

(59) Mac Connell, J. G., M. S. Blum and H. M. Fales: Science 168, 840 (1970).

(60) Rice, W. Y. and J. Y. Coke: J. Org. Chem. 31, 1010 (1965).

(61) Hegnauer, R.: Chemotaxonomie der Pflanzen: Basel, ab 1962 . Birkhäuser.

(62) Herbert, R. B. in: The Alkaloids (Chem. Soc. Spec. Rep.) Vol. VIl (M. F. Grundon et al. eds.), p. 4, London 1977, The Chem. Soc.

(63) Ritter, F. J., I. E. M. Rotgans, E. Talman, P. E. J. Verwiel and F, Stein: Experientia 29, 530 (1973). (64) Tursch, B., J. C. Braekman, D. Daloze, C. Hootele, D. Losman, R. Karlsson and J. M. Pasteels: Tetrahedron letters 201 (1973).

(65) Saxton, J. E. in: The Alkaloids (Chem. Soc. Spec. Rep.) Vol. IV, p. 114, London, 1974, The Chem. Soc. (66) Snieckus, V. A. in: The Alkaloids (Chem. Soc. Spec. Rep.) Vol. III, p. 322, London, 1973, The Chem. Soc.

(67) Snieckus, V. A. in: The Alkaloids (Chem. Soc. Spec. Rep.) Vol. V, p. 284, London, 1975, The Chem. Soc.

(68) Kem, R., B. C. Abbott, R. M. Coates: Toxicon 9, 15 (1971).

(69) Wheeler, J. W., O. Olubajo, C. B. Storm and R. M. Duffield: Science 211, 1051 (1981).

(70) Cavill, G. W. K. and E Houghton: Austr. J. Chem. 48, 228 (1974)

(71) Hagers Handb. der Pharmazeutischen Praxis, 4. Neuausgabe, (P. H. List und L. Hörhammer, eds.), Berlin, 1972, Springer.

(72) Zak. D. L., K. Ostovar and P. G. Keeney: J. Food Sci. 37, 967 (1972).

(73) Edgar, J. A., C. C. J. Culvenor and T. E. Pliske: Nature 250,646 (1974).

(74) Boppré, M.: Ent. exp. appl. 24, 264 (1978).

(75) Pliske, T. E.: Environm. Entomol. 4, 455 (1975).

(76) Mattocks, A. R. in: Phytochemical Ecology (J. B. Harborne, ed.), p. 179ff, London, 1972, Academic Press.

(77) Edgar, J. A., P. A. Cockrum and J. L. Frahn: Experientia 32, 1535 (1976).

(78) Benn, M., J. DeGrave, C. Gnanasunderam and R. Hutchins: Experientia 35, 731 (1979).

(79) Meinwald, J., C. J. Boriak, D. Schneider, M. Boppré, W. F. Wood and T. Eisner: Experientia 30, 721 (1974).

(80) Petty, R. L., M. Boppré, D. Schneider and J. Meinwald: Experientia 33, 1324 (1977)

(81) Boppré, M., R. L. Petty, D. Schneider and J. Meinwald: J. comp. Physiol. 126, 97 (1978).

(82) Goss, J. G.: Environm. Entomol. 8, 487 (1979).

(83) Boppré, M.: Biol. i. u. Zeit 7 161 (1977).

(84) Teas, H. J.: Biochem. Biophys. Res. Comm. 26, 686 (1967).

(85) Smith, J. N. in: Drug Metabolism from Microbe to Man (D. V. Parke and R. L. Smith, eds.), p. 226, London, 1977, Taylor and Francis Ltd.

(86) Jones, D. A., J. Parsons and M. Rothschild: Nature 193, 52 (1962). (87) Davis, R. H. and A. Nahrstedt: Comp. Biochem. Physiol. 64B, 395 (1979).

(88) Davis, R. H. and A. Nahrstedt: Comp. Biochem. Physiol, im Druck. (89) Nahrstedt, A. and R. H. Davis: Comp. Biochem. Physiol. 68B, 575 (1981).

(90) Conn, E. E.: Naturwiss. 66, 28 (1979).

(91) Nahrstedt, A. and R. H. Davis: in Vorber.

(92) Nahrstedt, A. und R. H. Davis: Planta med. 42, 124 (1981).

(93) Meinwald, J., Y. C. Meinwald, A. M. Chalmers and T. Eisner: Science 160,890 (1968).

(94) Hänsel, R.: Pharn. Ztg. 124 1054 (1979).

(95) Tschinkel, W. R.: J. Insect Physiol. 21, 753 (1974).

(96) Gregson, P. P., J. F. Marwood 
Münch. Ent. Ges. 67, 1 (1977) sowie pers. Mittlg.

(105) Blau, P. A., P. Feeny, L. Contardo, D. S. Robson: Science 200 , 1296 (1978).

(106) Schoonhoven, L. M. in: Insect Plant Relationships (H. F. van Emden, ed.), p. 87, Oxford, 1972, Blackwells Sci. Publ.

(107) Aplin, R. T., R. d'Acry Ward and M. Rothschild: J. Ent. (A) 50 73 (1975).

(108) Glombitza, K. W.: Struktur und biologische Bedeutung sekundärer Naturstoffe aus Meeresorganismen. Vortrag, Frühjahrstagung der Ges. für Biol. Chem., Münster, März 1980.

(109) Petty, R. L., M. Boppré, D. Schneider and J. Meinwald: Experientia 33,1324 (1977).

(110) Dettner, K., G. Schwinger: Biochem. Syst. Ecol. 8, 89 (1980).

(111) Honda, K.: J. Insect Physiol. $26,39(1980)$
(112) Duffey, S. S., M. S. Blum, H. M. Fales, S. L. Evans, R. W. Roncadori, D. L. Tiemann and Y. Nakagawa: J. Chem. Ecol. 3, 101 (1977). (113) Rothschild, M., R. Aplin, J. Baker and N. Marsh: Nature 280 , 487 (1979).

(114) Rüdiger, W.: Angew. Chemie $82,527(1970)$.

(115) Rüdiger, W, in: Pigments in Plants (F. C. Czygan, ed.), p. $314 \mathrm{ff}$ Stuttgart, 1980, G. Fischer.

(116) Eisner, T., L. B. Hendry, D B. Gekall and J. Meinwald: Science 172, 277 (1971).

(117) Bach, B., R. P. Gregson, G. S. Holland, R. J. Quinn and J. L. Reichelt: Experientia 34, 688 (1978).

(118) Swain, T.: Ann. Rev. Plant Physiol. 28, 479 (1979).

(119) Synge, L. R. M.: Naturwiss Rdsch. 24, 56 (1971).

(120) Lindley, M.: Nature 266, 776 (1977)

\section{Jüngere Monographien}

van Emden, H. T. (ed.), Insect Plant Relationships, Oxford, 1972, Blackwells Sci. Publ.

Harborne, J. B. (ed.), Phytochemical Ecology (Ann. Proc. Phytochem. Soc. Vol. 8), London 1972, Academic Press.

Wallace, J. W. and R. L. Mansell (eds.), Biochemical Interaction Between Plants and Insects (Rec. Adv. Phytochem. Vol. 10), New York, 1976, Plenum Press.

Sondheimer, E. and J. B. Simeone (eds.), Chemical Ecology, London 1976, Academic Press.

Harborne, J. B., Introduction to Ecological Biochemistry, London 1977, Academic Press.

Harborne, J. B. (ed.), Biochemical Aspects of Plant and Animal Coevolution (Ann. Proc. Phytochem. Soc. Vol. 15), London, 1978, Academic Press.

Rochstein, M. (ed.), Biochemistry of Insects, New York, 1979, Academic Press.

Adresse: Prof. Dr. A. Nahrstedt, Institut für Pharmazeutische Biologie, Mendelssohnstraße 1, D-3300 Braunschweig 